\title{
Factores que influyen en la sostenibilidad de las PyMES del sector restaurantero del municipio de Monterrey
}

\author{
Evelin Anahí Torres Torres y Alfonso López-Lira Arjona \\ Universidad Autónoma de Nuevo León
}

\begin{abstract}
The permanent closure of SMEs (small and medium-sized companies) is a relevant issue for the Mexican economy since SMEs represent $58 \%$ of the country's GDP (gross domestic product). It is important to identify the factors that influence SMEs to maintain business sustainability and prevent them from continuing to close. For this reason, the research to be carried out focuses on the theoretical proposal of the factors that allow increasing the sustainability of SMEs and thereby increasing or maintaining profitability, achieving it and thus avoiding the permanent closure of these companies. Therefore, the objectives of this article are to present the generalities of SMEs and theoretically support the factors proposed to achieve SME sustainability in Monterrey companies, then, risk control, the use of ICT (information and communication technologies), adaptive capacity, quality of service and knowledge management. Once the above factors have been supported, a questionnaire with a Likert scale is intended to measure the correlation between the independent and dependent variables.
\end{abstract}

Keywords: sustainability, profitability, SME.

\section{Resumen}

El cierre permanente de las PyMES (Pequeñas y Medianas Empresas) es un tema relevante para la economía mexicana puesto que las PyMES representan el $58 \%$ del PIB (Producto Interno Bruto) del país, entonces es importante identificar los factores que influyen en las PyMES para mantener una

\section{Factores que influyen en la sostenibilidad de las PYMES del sector restaurantero del municipio de Monterrey}


sostenibilidad empresarial y evitar que continúen cerrando. Por tal motivo, la investigación a realizar se enfoca en la propuesta teórica de los factores que permiten incrementar la sostenibilidad de las PyMES y con ello aumentar o mantener la rentabilidad logrando la permanecía y evitando así el cierre permanente de estas empresas. Los objetivos del presente artículo son presentar las generalidades de las PyMES y sustentar teóricamente los factores propuestos para lograr una sostenibilidad PyME en las empresas de Monterrey y se propone como factores el control de riesgos, el uso de las TIC's (Tecnologías de la Información y Comunicación), la capacidad adaptativa, la calidad en el servicio y la gestión del conocimiento. Una vez sustentados los factores anteriores se pretende realizar un cuestionario con escala Likert para medir la correlación entre las variables independientes y dependiente.

Palabras clave: sostenibilidad, rentabilidad, PyME. JEL: M19 043.

\section{Introducción}

La situación de las Pequeñas y Medianas Empresas en México es preocupante debido al cierre repentino que tienen aproximadamente el $80 \%$ de las PyMES, cifra que resulta alarmante dado que estas empresas son parte importante del país puesto que representan el $72 \%$ del empleo formal de todo el territorio mexicano, además de contribuir en aproximadamente el $58 \%$ del PIB de la economía del México al ser una problemática a resolver dado que la cuestión es evidente ya que se requiere identificar las causas de la ausencia de sostenibilidad en las PyMES. Las investigaciones en las que se identifica la sostenibilidad de las PyMES suelen orientarse a aspectos ambientales y no en aspectos internos y administrativos.

La dificultad en la esperanza de vida en las PyMES se debe a factores ausentes como cuestiones administrativas, liquidez, estructura tecnológica, conocimientos administrativos, capacitación en el recurso humano, la resistencia al cambio, los riesgos no identificados y la ausencia de elementos organizacionales. Dada la trascendencia derivada de la necesidad de incrementar la supervivencia de las empresas al hacer enfoque a cuestiones administrativas, la sostenibilidad es un objeto factible de estudio si se enfoca en la propuesta de nuevos modelos. 
De modo que investigar la sostenibilidad de las pequeñas empresas contribuye a eliminar la incertidumbre del porqué el $80 \%$ de las PyMES suelen cerrar sus puertas al público en un periodo tan corto. Se vuelve importante identificar los factores que permitan determinar la sostenibilidad de las Pequeñas y Medianas Empresas de Monterrey además de contribuir teóricamente en el avance del tema y su definición en el ámbito administrativo, generando mayor información de la sostenibilidad y las pequeñas empresas en Nuevo León. El resultado de la investigación podría permitir que menor cantidad de PyMES cierren y con ello aumentar o mantener la cantidad de empleos formales y la contribución del PIB en Nuevo León y también, según los resultados del análisis, podría servir de guía para próximas investigaciones en otros sectores del país, ya sea en otro estado o agregar diferentes factores que puedan estar relacionados con la sostenibilidad de las PyMES.

Entonces el objetivo principal de la investigación es identificar los factores que contribuyen en la sostenibilidad de las PyMES del municipio de Monterrey y específicamente los objetivos del estudio se basan en identificar la relación que tienen los factores como el control de riesgos, el uso de las tecnologías de la información y comunicación, la capacidad adaptativa, la calidad en el servicio y la gestión del conocimiento con la sostenibilidad de las PyMES del sector servicio.

Se plantea la siguiente hipótesis:

H1: los factores determinantes para la sostenibilidad de las Pequeñas y Medianas Empresas son el control de riesgos, uso de las Tecnologías de la Información y Comunicación, la capacidad de adaptación, la calidad en el servicio y la gestión del conocimiento.

\section{Fundamento de sostenibilidad PyME}

La sostenibilidad es un aspecto importante en todas las ramas de estudio. Aunque existe una gran cantidad de literatura sobre modelos de sostenibilidad a nivel social, la comprensión de los modelos de negocios sostenibles y cómo se "operacionaliza" el desarrollo sostenible en las empresas es débil. En administración, la sostenibilidad es un factor muy importante para la medición del desempeño ya que demuestra la viabilidad del negocio para mantenerlo y desarrollarlo al paso del tiempo (Sharma, Bhagwat y Dangayach, 2005).

\section{Factores que influyen en la sostenibilidad de las PYMES del sector restaurantero del municipio de Monterrey}


Los factores de sostenibilidad incluyen el crecimiento y las ganancias. No existen modelos teóricos unificados sobre el crecimiento de las empresas debido a la variedad de perspectivas e interpretaciones teóricas y empíricas, así como la complejidad innata del propio fenómeno del crecimiento que conlleva la sostenibilidad. Al estar inmerso el desempeño, la viabilidad y el crecimiento y la rentabilidad dentro de la sostenibilidad se pueden utilizar conceptos como desarrollo sostenible, sostenibilidad empresarial, desarrollo del negocio y éxito empresarial.

A pesar de los estudios existentes de la sostenibilidad uno de los mayores desafíos que se presenta es la ambigüedad en el concepto. La inexistencia de un consenso acerca del significado e implicaciones que tienen conceptos como sostenibilidad en la disciplina económica es un hecho innegable, por lo que se hace imperativo precisar en cada caso particular (para cada modelo y teoría) cuál es el significado que adopta (Olaya, 2006).

Entonces la idea de sostenibilidad se deriva del concepto de desarrollo sostenible que se convirtió en un lenguaje común en la primera Cumbre de la Tierra del Mundo en Río en 1987. El Informe Brundtland para la Comisión Mundial de Medio Ambiente y Desarrollo (1987) define la sostenibilidad como "desarrollo que se encuentra en el presente sin comprometer las capacidades de las generaciones futuras para satisfacer sus propias necesidades" (Brundtland, 1987).

La sostenibilidad es la forma en que una organización crea valor para sus propietarios y la sociedad maximizando los efectos positivos y minimizando los efectos negativos de los problemas sociales, ambientales y económicos. La idea de que la sostenibilidad tenga tres dimensiones puede vincularse a la Triple Línea de Fondo (TBL) que es un concepto de Elkington (1994). TBL incorpora tres dimensiones de desempeño: financiera, social y ambiental, y captura la esencia de la sostenibilidad al medir el impacto total de las actividades de una organización, incluida su rentabilidad y capital social y ambiental (Slaper y Hall, 2011).

La sostenibilidad empresarial o de la Pequeña y Mediana Empresa puede asemejarse a diversos conceptos administrativos como lo son la sostenibilidad empresarial, el desarrollo sostenible, desarrollo empresarial y la supervivencia empresarial y se describen los siguientes conceptos:

Torres Torres y López-Lira Arjona 
Sostenibilidad empresarial: la sostenibilidad empresarial puede explicarse como el proceso de gestión de una organización considerando tres aspectos diferentes: económica, social y ambiental (Slaper y Hall, 2011).

Desarrollo sostenible: Simon (1989) propone que la sostenibilidad es el eje central que une el entorno físico a la actividad humana local y a las políticas económicas que sugiere se podría dar por medio del desarrollo competitivo de las PyMES. Para que el desarrollo sea sostenible deben ser tomados en cuenta los factores sociales y los ecológicos, así como también los económicos sobre la base de los recursos vivos y no vivos y las ventajas y desventajas de las acciones alternativas en el largo y corto plazo (Galarza, Gómez y González, 2002).

Desarrollo empresarial o desarrollo de negocio: según Koontz y O'Donnell (2014) el desarrollo empresarial está relacionado con varios factores como crecer sobre la base de una unidad económica, crecer generando otras unidades económicas, mejorar la economía, eficiencia, efectividad, productividad, mejora continua y competitividad, también con la obtención de mejores niveles de liquidez, gestión, solvencia y rentabilidad.

Para alcanzar desarrollo empresarial es básico tener una adecuada gestión y que tiene que considerar al control como parte del proceso de gestión efectiva e indica que el control es la evaluación y corrección de las actividades de los subordinados para asegurarse de que lo que se realiza se ajusta a los planes.

La sostenibilidad PyME carece de una definición en conjunto, es decir, en la literatura se aprecian las definiciones de sostenibilidad y de PyME pero éstas en conjunto no se aprecian. Con lo anterior surge la variable Sostenibilidad PyME que hace referencia a la sostenibilidad de la Pequeña y Mediana Empresa, es decir, la conservabilidad de las PyMES.

Como se menciona en el párrafo anterior la sostenibilidad se ha definido por algunos autores en diversos enfoques y en su mayoría relacionados con el medio ambiente. En la presente investigación se requiere de una definición orientada a la administración que permite redactar su definición como a continuación se expresa para fines específicos de esta investigación.

Sostenibilidad PyME: proceso de gestión que se lleva acabo para mantener la rentabilidad organizacional de una empresa que se incluye a la organización y sus empleados con la finalidad de conservar la industria en

\section{Factores que influyen en la sostenibilidad de las PYMES del sector restaurantero del municipio de Monterrey}


situaciones de carencia logrando conservar el éxito, la innovación y el desarrollo del negocio.

Entonces se decidió elegir la definición propuesta por este estudio dado que abarca las dimensiones necesarias que guían y explican la orientación de la investigación, es decir, abarca al factor de la sostenibilidad dando a entender que se pretende conservar la industria en situaciones de carencia, haciendo referencia a conservar a la empresa aun en tiempos de crisis, además la definición se conjunta con las PyME que es otro factor importante dentro de la investigación ya que es el sector en el que se realiza la investigación, considerando esto una definición adecuada y que abarca los puntos suficientes para explicar y cubrir el objetivo de la investigación.

\section{Factores relacionados con la sostenibilidad PyME Control de riesgos}

El control de riesgos tiene implicaciones considerables para la competitividad y los negocios y permite el desarrollo de una estrategia para reducir las pérdidas potenciales mientras se aprovechan las áreas de oportunidad (Radner y Shepp, 1996). Además, la adecuada gestión de los riesgos aprueba que las empresas logren manejar el riesgo y la incertidumbre ya que aporta beneficios a la organización y se podría inferir que contribuye en mantener a la empresa en condiciones óptimas para lograr una supervivencia (Georges, 2013).

La incertidumbre de un evento, según las definiciones de autores $u$ organizaciones como IIA (2004), Keegan (2004) y COSO (2013), lo describen como el resultado a una acción que se pretende poner en marcha por la empresa, aceptando que los resultados pueden ser positivos o negativo. Una definición que considere al control de riesgos como una herramienta parece ser más útil ya que puede contemplar más elementos o pasos de algo tan amplio y profundo como lo son los riesgos que pueden afectar a la empresa además de preservar el valor de la organización.

De tal modo que al señalar la preservación del valor de una organización concuerda con la definición de sostenibilidad PyME que se propuso anteriormente y se concluye que la definición adecuada para la investigación es la propuesta por COSO debido a su relación entre ambas definiciones.

Algunos de los estudios relacionados con el control de riesgos son los publicados por Nunes y Serrasqueiro (2012), Senkus, Skrzypek, Luczak y

Torres Torres y López-Lira Arjona 
Malimowski (2014), Sharma, Bhagwat y Dangayach (2005), Bititci, Turner y Begemann (2000) y Drennan y McConnell (2007) se mencionan que para enriquecer a una organización se necesita controlar los riesgos a través de diversos indicadores que permitan evaluar la efectividad de la organización dado que es un requisito básico para tener éxito en un entorno que cambia dinámicamente.

Y por último Khudyakova et al. (2019) mencionan que la mayoría de los artículos científicos contemporáneos donde se estudia el control de riesgos señalan aspectos positivos sobre ella.

\section{Uso de las Tecnologías de Información y Comunicación}

En la actualidad gran parte de la sociedad tiene un amplio conocimiento sobre las Tecnologías de la Información y la Comunicación ya que éstas juegan un papel fundamental para el desarrollo de la sociedad, la economía y además para mejorar el nivel de crecimiento de las empresas, esencialmente para las Pequeñas y Medianas Empresas (PyMES) debido a las herramientas tecnológicas hoy se han convertido en una estrategia empresarial que necesitan las empresas para sobrevivir y mantenerse en su mercado (Maldonado-Guzmán, Pinzón-Castro y Rodríguez-González, 2020).

La Tecnología de la Información y Comunicación (TIC) está impregnando y transformando no sólo el ámbito empresarial sino todos los ámbitos de la vida, por lo cual se encuentra incluido el comercio, la educación, la salud, la gobernanza, la agricultura, la manufactura, la banca, la defensa, los medios de comunicación y el entretenimiento, la logística, los viajes y el turismo. Organizaciones como el Banco Mundial y el Banco Asiático de Desarrollo han señalado que las telecomunicaciones son ahora esenciales para el desarrollo económico y la competitividad de un país. En un informe del Banco Mundial se ha descubierto un aumento del $10 \%$ en la velocidad de la conexión a Internet de alta velocidad amplía el crecimiento económico en un 1,3\% (Minges, 2016).

Según Gargallo y Pérez (2009) las Tecnologías de la Información y Comunicación son la posibilidad que tienen las organizaciones de ampliar sus oportunidades como facilitar la expansión de productos, la mejora del servicio al cliente, la adaptación y respuesta a la demanda, así como la mejora de las relaciones con clientes y proveedores para conseguir mejoras en su posición

\section{Factores que influyen en la sostenibilidad de las PYMES del sector restaurantero del municipio de Monterrey}


competitiva de mercado.

Entonces la definición anterior se adecúa a la investigación porque toma en cuenta la necesidad que tienen las empresas para requerir tecnología en pos del crecimiento y desarrollo organizacional, además de tomar en cuenta la expansión de oportunidades y para mejorar el nivel de crecimiento de las PyMES a través del uso adecuado de las tecnologías. $Y$ aunque las otras definiciones son precisas no están conceptualizadas más allá del uso técnico e inmediato de las Tecnologías de la Información y Comunicación que provoca no expliquen el concepto en el contexto que se requiere para la presente investigación.

El uso de las TIC's representa un factor a tomar en cuenta al momento de hablar de sostenibilidad PyME dado que como menciona Porter (1980, citado en Maguire, Koh y Magrys, 2007): el uso de las TIC's permite generar una ventaja competitiva ante la competencia y por ende contribuye en la supervivencia de la empresa.

Autores como Bardhan, Whitaker y Mithas (2006), Díaz-Chao y TorrentSellens (2010) comparten la idea de Porter (1980), dado que el uso de las TIC's son una estrategia que permite a las empresas mejorar la competitividad en su interés particular en mejorar sus sistemas de trabajo. Asimismo, algunas investigaciones empíricas como las de Bhatt y Grover (2005) y Mithas Ramasubbu, Krishnan y Sambamurthy (2005) han demostrado que las inversiones en tecnologías de la información y las capacidades a través de las TIC's están asociadas con una mayor productividad con la satisfacción del cliente con capacidad organizativa y desempeño de las empresas.

Entonces la relación existente entre las TIC's y la sostenibilidad de la PyME se ve relacionada con la sostenibilidad de las empresas debido al éxito que se alberga en las TIC's y las PyMES logrando brindar una ventaja competitiva. Además, algunas investigaciones realizadas en empresas estadounidenses en los años 90 obtuvieron contribuciones positivas de las TIC's en la productividad de las empresas que las adoptaban y productos marginales brutos superiores a los correspondientes al resto de capital. Algunos estudios fueron publicados por autores como Brynjolfsson (1993), Bresnahan, Brynjolfsson y Hitt (1999), Lichtenber (1995) y Gargallo-Castel y Pérez-Sanz (2009). 


\section{Capacidad de adaptación}

Es oportuno mencionar la definición de capacidades dinámicas para entender adecuadamente la capacidad de adaptación. Teece, Pisano y Shuen (1997) definen las capacidades dinámicas como "la capacidad de la empresa para integrar, construir y reconfigurar competencias internas y externas para abordar entornos que cambian rápidamente". El propósito de las capacidades dinámicas es lograr la estabilidad renovando, recombinando, redistribuyendo, replicando, reduciendo y retirando recursos o capacidades en un entorno empresarial volátil hacia ganancias comerciales.

Idealmente los países en desarrollo evolucionan a partir de capacidades, procesos o prácticas comerciales (Faizal, Zaidi y Othman, 2012). Wang y Ahmed (2007) identifican tres dimensiones de los países en desarrollo: capacidad de adaptación, capacidad de absorción o capacidad innovadora.

Se optó por estudiar la capacidad de adaptación ya que la literatura indica que es un proceso de cambio organizacional que debe practicarse en el contexto de un proceso de continuidad de mejoramiento humano y organizacional en el tiempo. Esto permite que una organización y su gente se adapten eficazmente al cambio del entorno, entonces se considera adecuado para estudiarlo en las pequeñas empresas ya que como se sabe se encuentran en un entorno ampliamente dinámico (Shane y Venkataraman, 2000).

Algunas definiciones para comprender mejor lo que es la capacidad de adaptación se enlistan a continuación: según Jean Piaget la adaptación debe describirse como un equilibrio entre la acción del organismo sobre el medio ambiente y viceversa. En el ámbito de la administración se toma como el equilibro entre las acciones de la empresa sobre y un entorno dinámico (2003).

Posteriormente Lockett, Wiklund, Davidsson y Girma (2011) mencionan que la adaptabilidad también se puede describir en términos de la competencia de la empresa para alterar su comprensión de las expectativas del mercado, esto aumenta como resultado del aumento de los límites de las empresas (Lockett et al., 2011). Y Busquets, Rodon y Wareham (2009) agregan que la adaptabilidad como la capacidad de cambiar consciente o inconscientemente para adaptarse a diferentes circunstancias como cambios en el entorno.

Las definiciones anteriores coinciden en que la adaptabilidad organizacional es una capacidad de la empresa para adaptarse a un ambiente

\section{Factores que influyen en la sostenibilidad de las PYMES del sector restaurantero del municipio de Monterrey}


externo. La definición de Busquets, Rodon y Wareham se diferencia de entre las demás establecidas dado que ésta se adapta a diferentes circunstancias, significa que su definición no considera específicamente el entorno externo sino que también está implícito el entorno interno de la organización.

Entonces es conveniente elegir la definición de Busquets, Rodon y Wareham para el estudio ya que concuerda con la definición elegida de sostenibilidad PyME y además es relevante tomar en cuenta que la definición involucra en su redacción las diferentes circunstancias y es precisa dado que en ocasiones existen circunstancias en las que se debe adaptar al ambiente interno de una empresa. Un ejemplo es el escenario donde se adquieren nuevas tecnologías y se necesita capacitar al personal en su uso, es decir, adaptarse a ellas de tal modo que se evolucione hacia el cambio organizacional.

La capacidad de adaptación se ha estudiado en ámbitos empresariales relacionados con el éxito de las empresas, la supervivencia empresarial, el desempeño y el desarrollo empresarial donde señalan el valor de este factor en las empresas, de igual forma lo menciona Pérez (2009) en la capacidad de adaptación como un factor importante para la supervivencia de las organizaciones en el largo plazo va a depender de sus respectivos éxitos para adecuarse a un entorno caracterizado por el continuo cambio. Siendo así las capacidades de absorción, innovación, aprendizaje y adaptación han sido propuestas por la literatura como las habilidades idóneas para alterar los recursos organizacionales con el fin de desarrollar estrategias que les generen valor (Pérez, 2009).

El éxito en los entornos dinámicos causado por la frecuente renovación de estrategias como el que se presenta en las PyMES requiere de adquirir nuevos conocimientos. La adaptación se vuelve un factor vital e irremplazable para lograr sobrevivir en entornos turbulentos (Cruz, Navas, López y Delgado, 2009).

Winter (2003), Zahra, Sapienza y Davidsson (2006) y Danneels (2008) mecionan que la capacidad de adaptación de la empresa a entornos dinámicos debe entenderse como una de las principales capacidades dinámicas ya que consiste en la capacidad de generar nuevas capacidades y nuevo conocimiento como un elemento imprescindible para la supervivencia de la empresa ante regímenes de rápido cambio (Teece, Pisano y Shuen, 1997).

Torres Torres y López-Lira Arjona 


\section{Calidad en el servicio}

Para Deming (1989) la calidad se define en función del sujeto o cliente y se traduce como las necesidades futuras del cliente a características evaluables para que el producto logre ser diseñado y fabricado y proporcione satisfacción por un precio que el cliente esté dispuesto a pagar.

Tuchman (1980) sostiene que la calidad es la inversión de mejor habilidad y esfuerzo para producir mejores y más admirables resultados.

Para Dale, Van der Wile y Iwaarden (2007) la definición se reduce a que la calidad en el servicio se basa en el cumplimiento de los requisitos y las especificaciones que satisfagan y deleiten al cliente.

Zeithaml (1988) menciona que la calidad puede ser definida en términos generales como la superioridad o excelencia de un producto o servicio.

Las definiciones anteriores explican la calidad del servicio de una forma adecuada. Para la investigación presente se tomará la definición propuesta por Dale, Van der Wile y Iwaarden (2007) ya que es muy clara porque precisa que la calidad en el servicio requiere cumplir con los requisitos del cliente a diferencia de las demás definiciones que se enfocan en la calidad de un producto, lo que evita que sean elegidas para la investigación ya que ésta se orienta en la calidad en el servicio de los clientes en el área de PyMES restauranteras.

En las investigaciones de Berry y Parasuraman en 1988 se mencionó que para medir la calidad del servicio era necesario tomar en cuenta 10 dimensiones. Años más tarde encontraron que no se requiere el estudio de las 10 dimensiones para lograr medir la calidad del servicio y se redujo la cantidad a 5 dimensiones (fiabilidad, tangibilidad, responsabilidad, garantía y seguridad). En la presente investigación se utilizaron únicamente 4 dimensiones (excluyendo la dimensión de garantía) ya que la dimensión de garantía es irrelevante en el contexto de las PyMES restauranteras, además en las investigaciones donde han utilizado estas dimensiones existe una falta de consenso sobre su definición y operacionalización (Zeithaml, Parasuraman y Berry, 1988; Berry y Parasuraman, 2004; Cook y Rohit, 2002; Zeithaml, Parasuraman y Berry, 1985; Steenkamp, 1990; Grönroos, 1994).

\section{Factores que influyen en la sostenibilidad de las PYMES del sector restaurantero del municipio de Monterrey}




\section{Gestión del conocimiento}

Andreu y Siever (1999) definen la gestión del conocimiento como "el proceso que continuamente asegura el desarrollo y aplicación de todo tipo de conocimientos pertinentes en una empresa, con el objetivo de mejorar su capacidad de resolución de problemas y así contribuir a la sostenibilidad de sus ventajas competitivas".

Beckman (1997) define que "la gestión del conocimiento es la formalización y el acceso a la experiencia, el conocimiento y la experiencia que crean nuevas capacidades, permiten un desempeño superior, fomentan la innovación y mejoran el valor del cliente".

Alavy y Leidner (2001) definen gestión de conocimiento en organizaciones como "aquella que se refiere a la identificación y el aprovechamiento del conocimiento colectivo en una organización para ayudar a la organización competir, en el marco de tres procesos básicos de creación, almacenamiento y recuperación, transferencia y aplicación del conocimiento".

Para la elección de una definición de gestión del conocimiento adecuada para la investigación se tomó en cuenta el contexto de ésta: las PyMES del sector servicio de la industria restaurantera. Si bien todas las definiciones coinciden en que es importante la creación, transmisión y aplicación del conocimiento, la definición de Alavy y Leidener destaca por el aprovechamiento colectivo en una organización con la finalidad de competir, trata de explicar que se extrae lo mejor de cada persona que labore en la organización con la finalidad de utilizarlo y explotar los conocimientos en donde mejor convenga.

La calidad en el servicio se conforma por 3 dimensiones que son: la creación del conocimiento, almacenamiento y transferencia del conocimiento junto la aplicación del conocimiento, que según los autores Huang y Shih (2009), Rollett (2003), Alavy y Leidner (2001) y Armistead (1999) es la manera idónea para mediar la variable ya que permiten evaluar la amplitud del constructo.

Varios autores coinciden en el argumento sobre el panorama competitivo global actual ya que el conocimiento y la información constituyen condicionantes claves de la rentabilidad y supervivencia de las empresas en mayor medida que puedan ser los factores productivos tradicionales como la tierra, el capital o el trabajo, así hay algunos autores (Hall, 1993; Grant, 1996; Zahra, 1999; Dean y Kretschmer, 2007) que han investigado que la gestión del 
conocimiento influye en la sostenibilidad de las empresas.

\section{Métodos}

La investigación que se plantea es de tipo no experimental ya que las variables serán será de tipo cuantitativa, exploratoria, descriptiva, correlacional y explicativa, será exploratoria porque se examinará un tema que ha sido estudiando pocas veces e identificarán factores que permitirán un análisis profundo. Correlacional porque se buscará conocer cómo se puede comportar un concepto o variable al conocer el comportamiento de otra.

Se opta por identificar la presente investigación como descriptiva porque se busca describir la relación que existe entre las variables, es decir, la base del estudio correlacional que se hará y es explicativa porque se detallará información entre la relación de dos variables o conceptos (Pimienta Prieto y De la Orden Hoz, 2012).

Y se pretende utilizar como población a las PyMES que se encuentran en la Zona Metropolitana de Monterrey al tomar una muestra probabilística a conveniencia. Posteriormente se analizará la información mediante la estadística descriptiva y haciendo uso del programa estadístico SPSS 25.

\section{Conclusiones}

Las variables independientes elegidas para conformar el modelo de investigación se deben al historial empírico que tienen sobre el éxito de las PyMES, es decir, esta investigación busca generar un modelo que permita establecer una sostenibilidad de las PyMES con relación en que ésta logre sobrevivir en tiempos de carencia o permanecer en entornos dinámicos y a pesar de las circunstancias continúen con éxitos y permanezcan en el mercado desarrollando sus capacidades como lo podría ser el control de riesgos, la capacidad de adaptación y el adecuado uso de las TIC's.

Se pretende probar si efectivamente las variables elegidas para este modelo son factores que realmente impactan en la sostenibilidad de las Pequeñas y Medianas Empresas del municipio de Monterrey. De esta manera se plantean las siguientes hipótesis de investigación:

H2: identificar si el control de riesgos es un factor relacionado positivamente con la sostenibilidad de las PyMES del sector servicio del municipio de Monterrey.

\section{Factores que influyen en la sostenibilidad de las PYMES del sector restaurantero del municipio de Monterrey}


H3: identificar si el uso de las Tecnologías de la Información y Comunicación es un factor relacionado positivamente con la sostenibilidad de las PyMES del sector servicio del municipio de Monterrey.

H4: identificar si la capacidad de adaptación es un factor relacionado positivamente con la sostenibilidad de las PyMES del sector servicio del municipio de Monterrey.

H5: identificar si la calidad en el servicio es un factor relacionado positivamente con la sostenibilidad de las PyMES del sector servicio del municipio de Monterrey.

H6: identificar si la gestión del conocimiento es un factor relacionado positivamente con la sostenibilidad de las PyMES del sector servicio del municipio de Monterrey.

El conjunto de variables independientes utilizadas en el modelo de esta investigación es un modelo que pretende reconfigurar a las empresas y brindar posibilidad de generar nuevas habilidades para permanecer en el mercado y mantener estabilidad o crecimiento en la rentabilidad de las empresas, además lograr una sostenibilidad empresarial o sostenibilidad de la PyME como se indica en el modelo gráfico de la investigación. 


\section{Referencias}

Anderson, E. W., Fornell, C. y Lehmann, D. R. (1994). Customer satisfaction, market share, and profitability: findings from sweden. Journal of marketing, 53-86.

Anderson, E. W., Fornell, C. y Rust, R. T. (1977). Customer satisfaction,productivity, and profitability: differences between goods and service. Marketing science, 129-145.

Bititci , U. S., Turner, T. y Begemann, C. (2000). Dynamics of performance measurement systems. International Journal of Operations and Production Management, 692-704.

Bombiak, E. (2020). Advances in the implementation of the model of sustainable human resource management: polish companies' experiences. Entrepreneurship and sustainability issues, 1667-1687.

Brundtland, G. (1987). Our common future: the world commission on environment and development. Oxford: Oxford University Press. https://www.un.org/es/ga/president/65/issues/sustdev.shtml

Brustbauer, J. (2014). Enterprise risk management in SMEs: towards a structural model. International Small Business Journal, 1-16.

Busquets, J., Rodon, J. y Wareham, J. (2009). Adaptability in smart business networks: an exploratory case in the insurance industry. Decision Support Systems, 287-296.

Chakravarthy, B. (1982). Adaptation: a Promising Metaphor for Strategic Management. Academy of Management Review, 35-44.

Chege, S. M., Wang, D. y Suntu, S. L. (2019). Impact of information technology innovation on firm performance in Kenya. Information Technology for Development.

Cobo-Romaní, J. C. (2011). El concepto de tecnologías de la información. Benchmarking sobre las definiciones de las TIC en la sociedad del conocimiento. Zer-Revista de Estudios de Comunicación, 295-318.

COSO. (2013). Committe of Sponsoring Organizations of the Treadway Commission. Committe of Sponsoring Organizations of the Treadway Commission. https://www.coso.org/Pages/ic.aspx

Cruz, J., Navas, J., López, P. y Delgado, M. (2009). Concepto e implicaciones de las capacidades dinámicas desde un enfoque de dirección del conocimiento. AECA.

\section{Factores que influyen en la sostenibilidad de las PYMES del sector restaurantero del municipio de Monterrey}


Delmar, F., Davidsson, P. y Gartner, W. (2003). Arriving at the High-Growth Firm. Journal of Business Venturing, 189-216.

Djordjevic, A. y Cotton, D. (2011). Communicating the sustainability message in higher education institutions. International Journal of Sustainability in Higher Education, 381-394.

Domańska-Szaruga, B. (2020). Maturity of risk management culture. Entrepreneurship and sustainability issues, 7(3), 2060-2078.

Doms, M., Dunne, T. y Roberts, M. J. (1995). The role of technology use in the survival and growth of manufacturing plants. International Journal of Industrial Organization, 523-542.

Drennan, L. T. y McConnell, A. (2007). Risk and Crisis Management in the Public Sector. Routledge.

Drucker, P. F. (1995). Innovation \& Entrepreneurship. Harper Business, 28.

Eikelenboom, M. y De Jong, G. (2019). The impact of dynamic capabilities on the sustainability performance of SMEs. Journal of Cleaner Production, 1360-1370.

Eisenhart, K. M. y Martin, J. A. (2000). Dynamic capabilities: what are they? Strategic Managment Journal, 1105-1121.

Elkington, J. (1994). Towards the Sustainable Corporation: Win-Win-Win Business Strategies for Sustainable Development. California Management Review, 90-100.

Eze, S., Duan, Y. y Awa, H. O. (2013). Dynamic capabilities approach to information communication technology adoption in SMEs. UK Academy for Information Systems Conference Proceedings.

Faizal, M., Zaidi, A. y Othman, S. N. (2012). Understand the concept of dynamic capabilities by dismantling Teece, Pisano, and Shuen (1997) definition. International Journal of Academic Research in Business and Social Sciences, 367-378.

Fansuri, M. (2019). The Role of Entrepreneurial Orientation and Adaptive Capability to Performance of SME Food \& Beverages. Global Business \& Management Research , 139-151.

Fornell, C. (1992). A national customer satisfaction barometer: The swedish experience. Journal of marketing, 6-21.

Galarza, E., Gómez, R. y Gonzalez, L. (2002). Ruta hacia el desarrollo sostenible del Perú: Lima. Centro de Investigación de la Universidad del Pacífico, 11. 
Gargallo-Castel, A. y Pérez-Sanz, J. (2009). El papel de las Tecnologías de la Información y la Comunicación en las empresas de economía social. Revesco, 90-116.

Georges, D. (2013). Risk management: history, definition and critique. Risk Management and Insurance Review, 147-166.

Giarratana, M. S. y Torrisi, S. (2010). Foreign entry and survival in a knowledgeintensive market: emerging economy countries' international linkages, technology competences, and firm experience. Straregic Entrepreneurship Journal, 85-104.

Gremler, D. y Gwinner, K. P. (2000). Customer-employee rapport in service relationships. Journal of service research, 82-104.

Grzeszczyk, T. A. y Waszkiewicz, M. (2020). Sustainable investment project evaluation. Entrepreneurship and sustainability issues, 2363-2381.

Hartoyo, H. y Daryanto, H. (2016). The effects of ICT adoption on marketing capabilities and business performance of Indonesian SMEs in the fasion industry. Journal of Business and Retail Management Research, 2-17.

Høgevold, N. M. y Goran, S. (2012). A business sustainability model: A European case study. Journal of Business \& Industrial Marketing, 142151.

Hopkin, P. (2010). Fundamentals of risk management. United States: Kogan Page Limited.

IIA. (2004). Institute of Internal Auditors. Institute of Internal Auditors. www.theiia.org

INEGI. (2018). INEGI. https://www.gob.mx/se/prensa/inegi-presentaresultados-de-la-encuesta-nacional-sobre-productividad-ycompetitividad-de-las-mipymes-enaproce-2018-215878

Institute of Risk Management. (2002). A risk Management Standard. www.theirm.org.

Keegan, M. (2004). The orange book. Londres: H.M. Treasury.

Khudyakova, T., Shmidt, A. y Shmidt, S. (2019). Implementation of controlling technologies as a method to increase sustainability of the enterprise activities. Entrepreneurship and Sustainability Issues, 1185-1196.

Kihara, P., Bwisa, H. y Kihoro, J. (2016). Relationships among Structural Adaptations, Strategy Implementation and Performance of

\section{Factores que influyen en la sostenibilidad de las PYMES del sector restaurantero del municipio de Monterrey}


Manufacturing Small and Medium Firms Tinhika, Kenya. British Journal of Applied Science \& Technology, 1-16.

Koontz, R. y O'Donnell, C. (2014). Administración Moderna. Ciudad de México: Litografía Ingramex S.A.

Lockett , A., Wiklund, J., Davidsson, P. y Girma, S. (2011). Organic and acquisitive growth. Journal of Managment Studies , 217-226.

Maguire , S., Koh, S. y Magrys, A. (2007). The adoption of e-business and knowledge management in SMEs. Benchmarking: an International Journal, 37-58.

Maldonado-Guzmán, G., Pinzón-Castro, S. Y. y Rodríguez-González, R. M. (2020). The impact of information and communication technology in mexican SMEs growth. Advances in Management \& Applied Economics.

Meraz-Ruiz, L. (2014). Estrategias de Competitividad de las Micro, Pequeñas y Medianas Empresas Vinícolas de la ruta del vino del Valle de Guadalupe, Baja California, México. México: Baja California.

Minges, M. (2016). World Bank. World Bank. http://documents1.worldbank.org/curated/en/178701467988875888/p df/102955-WP-Box394845B-PUBLIC-WDR16-BP-Exploring-theRelationship-between-Broadband-and-Economic-Growth-Minges.pdf

Naldi, L., Nordqvist, M., Sjoberg, K. y Wiklund, J. (2007). Entrepreneurial Orientation, Risk Taking, and Performance in Family Firms. Family business review, 20(1), 33-47.

Nguyen, N. H., Beeton, R. J. y Halog, A. (2016). Firm characteristics and its adaptive capacity in response to environmental requirements: an empirical study of Vietnam's textile and garment SMEs. International Journal of Environment and Sustainability.

Nunes, P. M. y Serrasqueiro, Z. (2012). Are young SMEs' survival determinants different? Empirical evidence using panel data. Applied Economics Letters, 849-855.

OCDE. (2002). Organización para la Cooperación y el Desarrollo Económicos. http://www.oecd.org/dataoecd/3/8/20627293.pdf

Olawale, F. (2019). Sustainability orientation and sustainable entrepreneurial intentions of university students in south Africa. Entrepreneurship and sustainability issues, 990-999. 
Olaya, Á. P. (2006). Los modelo neoclásicos de desarrollo sostenible y la noción de "sostenibilidad débil". Revista científica Guillermo de Ockham, 4(1).

Ongori, H. y Migiro, S. O. (2010). Information and communication technologies adoption in SMEs: Literature review. Journal of Chinese Entrepreneurship, 93-104.

Pérez, M. (2009). La relación entre la tecnología de la información, las prácticas de gestión de la calidad y el desarrollo de las capacidades dinámicas: una aproximación empírica. [Tesis Doctoral]. Universidad de Granada.

Piaget, J. (2003). The psychology of intelligence. Routledge: Taylor and Francis e-library .

Pimienta Prieto, J. H. y De la Orden Hoz, A. (2012). Metodología de la investigación. México: Pearson Educación.

Porter, M. (1980). Competitive strategy: techniques for analyzing industries and competitors. New York: Free Press.

Radner, R. y Shepp, L. (1996). Risk vs. profit potential: a model for corporate strategy. Journal of Economic Dynamics \& Control, 1373-1393.

Rogers, E. M. y Allbritton, M. M. (1995). Interactive Communication Technologies in Business Organizations. Journal of Business Communication, 177-195.

Rolstadas, A. (1998). Enterprise performance measurement. International Journal of Operations \& Production Management, 989-999.

Rose, R. C., Kumar, N. y Li yen, L. (2006). Entrepreneurs success factors and escalation of small and medium sized enterprises in Malaysia. Journal of Social Sciences, 2(3), 74-80.

Senkus, P., Skrzypek, A., Luczak, M. y Malimowski, A. (2014). The model of an organization performance measurement in the context of sustainable system management. Administracja i Zarzqdzanie, 163-172.

Shane, S. y Venkataraman, S. (2000). The promise of entrepreneurship as a field of research. Academy of Management Review, 217-226.

Sharma, M. K., Bhagwat, R. y Dangayach, G. S. (2005). Practice of performance measurement: experience from Indian SMEs. International Journal of Globalization and Small Business, 183-213.

\section{Factores que influyen en la sostenibilidad de las PYMES del sector restaurantero del municipio de Monterrey}


Slaper, T. F. y Hall, T. J. (2011). Slaper, T. F. and The triple bottom line: what is it and how does it work? Indiana Business Review, 4-8.

Stefanovic, I., Prokic, S. y Rankovic, L. (2010). Motivational and success factors of entrepreneurs: the evidence from a developing country. Journal of Economics and Business, 251-269.

Stubbs, W. y Cocklin, C. (2008). Conceptualiziing a "Sustainability Business Model". Organization \& Environment, 103-127.

Svensson, G. y Wagner, B. (2011). Transformative business sustainability: Multi-layer model and network of e-footprint sources. European Business Review, 23, 334-352.

Teece, D., Pisano, G. y Shuen, A. (1997). Dynamic Capabilities and Strategic Management. Strategic Management Journal, 18(7), 509-553.

Turlakova, S. S. (2019). Information and communication technologies for the development of "smart" industries. Economy of Industry, 101-122.

UNESCO. (2002). Records of the General Conference. París: United Nations Educational, Scientific and Cultural Organization.

Wang, C. L. y Ahmed, P. K. (2007). Dynamic capabilities: a review and research agenda. The international journal of management reviews, 31-51.

Zhao, Y. L. y Di Benedetto, C. A. (2013). Designing service quality to survive :empirical evidence from chinese new ventures. Journal of business research, 66(8), 1098-1107.

Zhou, K. Z. y Li, C. B. (2010). How strategic orientations influence the building of dynamic capability in emerging economies. Journal of Business Research, 224-231. 